\title{
The Potential and Risks of Internet Use as Permitted by Jewish Law Case Study: Internet Use by Students of a Religious High School - by Default or by Choice?
}

\author{
Nitza Davidovitch \\ 'Ariel University, Israel \\ Email:d.nitza@ariel.ac.il Tel:972-3-9066103
}

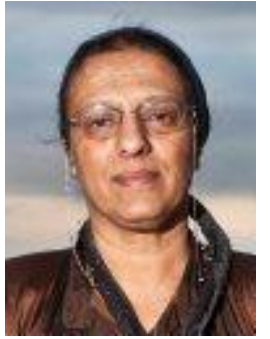

\begin{abstract}
Google is celebrating its $20^{\text {th }}$ anniversary - and the internet has become an inseparable part of the lives of adults, teens, and children. In awareness of the problems and challenges posed by the new world, various software programs have been developed, among them NetSpark (henceforth: the program), which make it possible to block and/or filter information received from the web (Wells and Lewis, 2006). In several Israeli schools, the management has decided to install a filter in their students' cellular devices in order to maintain safe surfing even after school hours. The option of blocking or filtering websites on school computers existed previously, but the innovation offered by the current program is the possibility of screening inappropriate content on students' smartphones. The study examined the association between installing the program on smartphones and utilization of leisure time among 120 female high school students, half of whom used the program. The association between use of the program and the students' sociodemographic background, smartphone use patterns, and utilization of leisure time was examined. Research findings show no difference between those who use the program and those who don't in the association between use of smartphone apps and internet surfing, utilization of leisure time, duration of internet use, and duration of cellular phone use. However, use of cellphone apps was higher among respondents who ranked themselves less religious than among respondents who ranked themselves more religious. The significance of the findings for parents and educators is that internet use is an issue that requires attention among students in religious high schools as well. The question is whether supervision should be imposed through the use of filters or should youngsters be taught how to cope with the global world.
\end{abstract}

Keywords: IoO, I3 1, I 39.

Citation | Nitza Davidovitch (2018). The Potential and Risks of Internet Use as Permitted by Jewish Law Case Study: Internet Use by Students of a Religious High School - by Default or by Choice? Journal of Education and e-Learning Research, 5(2): 96-101.

History:

Received: 12 March 2018

Revised: 29 March 2018

Accepted: 2 April 2018

Published: 4 April 2018

Licensed: This work is licensed under a Creative Commons

Attribution 3.0 License (c))

Publisher:Asian Online Journal Publishing Group
Funding: This study received no specific financial support.

Competing Interests: The author declares that there are no conflicts of interests regarding the publication of this paper.

Transparency: The author confirms that the manuscript is an honest, Transparency: The author confirms that the manuscript is an honest,
accurate, and transparent account of the study was reported; that no vital features of the study have been omitted; and that any discrepancies from the study as planned have been explained.

Ethical: This study follows all ethical practices during writing.

\section{Contents}

1. Introduction

2. Method

99

3. Results

4. Conclusion and Discussion. 100
101

References. 


\section{Introduction}

In recent years, the internet has become a major sphere of activity for children and teens, for purposes of information, interpersonal communication, entertainment and social needs. On the internet teens listen to music, play computer games, search for information, talk to their friends, write about themselves, portray their photographs, get to know new people, respond to posts, and more. In this way, the virtual world has become an inseparable part of the physical world of children and teens (Almog, 2004). Hence, the time they spend facing the computer screen is growing. A survey conducted in the US found that children and teens devote about two and a half hours a day to computer use; in Europe 60\% of 9-16 years, olds were found to use the internet every day or almost every day, for 90 minutes on average. In Israel, $92 \%$ of 13-17 years olds surf the web about two hours a day on average (Sasson et al., 2011; Israel Internet Association, 2014).

With the advantages of the internet has come an awareness of its disadvantages. Problematic internet use might lead to exposure to age-inappropriate contents, mental distress, and addiction. Theories dealing with health risks (Sasson et al., 2011) show that the perception of a behavior as dangerous is an important component in reducing engagement in this behavior. Moreover, in adolescence, there is a rise in the significance of the peer group and it has a growing influence on the behavior of individuals. Therefore, it is important to understand the outlook of children and teens in order to successfully construct effective prevention programs to reduce risky behaviors on the internet.

In Israel, internet use is a particularly challenging issue within religious society. Many studies indicate that in the religious sector, the issue of leisure and its risks is all the more acute (Bar-Lev, 1977;1981; Ben Zvi, 1994; Brandes, 2011; Gopes, 2011).

\subsection{Use of the Internet}

In 2000, the office of President Bill Clinton published the report "Falling through the net: Defining the digital divide", which stated that over $50 \%$ of households in America have a computer and that access to the internet had grown significantly, from $26.2 \%$ in 1998 to $41.5 \%$ in 2000 . Furthermore, global internet use had grown rapidly to approximately 581 million users in 2002 , a rise of 463 million from the previous year. The more this electronic medium continues to expand, we can be expected to see a rise in problem behaviours associated with its implementation.

Problematic internet use may be manifested in computer addiction, internet addiction disorder, internet mania, and pathological internet use. Moreover, reports by many people with internet use problems have been published in recent years on the popular media and in the medical literature. Above all, problematic internet use might be categorized as an individual's inability to control his or her internet use, leading to distress and dysfunction in daily activities.

Many potential dangers are related to internet use and overuse. Problematic internet use is known as one's inability to control his or her internet use. The change causes stress and/or functional failure and has been described in the psychological literature as an addiction to the internet and pathological internet use. Based on the fifth edition of the DSM, the definition is one of clear pathological dependency (Shapira et al., 2000). The internet might serve as a platform for a psychiatric illness (pathological addiction, paraphilia - deviant sexual behavior, and obsessive purchasing). Small systematic face-to-face psychiatric studies of people with internet use problems suggest high rates of comorbidity, including moods and anxiety disorders. Studies show that problematic internet use is also related to significant distress and dysfunction (Shapira et al., 2003). People with problematic internet use have reported high depressive symptoms. An online survey found depressive symptoms among 259 people classified as having an internet use problem. In addition, a study with 169 participants found an association between massive internet use and depression and loneliness, reduced contact with family members, and social deterioration (Shapira et al., 2000).

\subsection{Information on Smartphone Use}

Today, smartphones are considered more than merely mobile phones. Thanks to the extensive functions they offer, smartphones are not only a means of communication, but also a real-time source of information. In addition, they provide comfort in people's daily lives and in many cases, are also related to patterns of addiction.

While the adult population has "migrated" to the online environment, teens are growing up today in an era where the existence of the internet and its use are perceived as almost inherent. Moreover, they are digital natives who grew up surrounded by smartphones and the smartphone has become integrated in their lifestyle and identity. Therefore, teens more than adults are expected to be internet users. This assumption was confirmed in a study by the Pew Institute on intergenerational differences with regard to internet use among Americans. Hence, it is no surprise that in Israel as well survey findings show a similar disparity, with $86 \%$ of teens using the internet versus $68 \%$ of adults (Rafaeli et al., 2010; IIA, 2014).

Smartphones have become a nearly inseparable part of the daily life of young Israelis. A survey conducted in 2015 found that the average age in which children are first given a smartphone is 9.5. Further, the average duration of daily use at ages 12-17 is 3.4 hours. Girls were found to use smartphones more than boys. With regard to use of applications installed on the phone, $80 \%$ of $13-17$ years olds were found to use the WhatsApp message app for daily communication, 72\% of Israeli teens use Instagram, and 55\% Snapchat (Lenhart, 2015). A study conducted at the University of Haifa showed that $94 \%$ of high school students in Israel access social networks in class. Only $4 \%$ make no use of smartphones during class. The researchers stated that the students use their mobile phones in various ways - to surf the web and social networks, listen to music, take pictures, play games, and send text messages and photographs. According to the survey findings, there is almost no moment during class in which no students are using a smartphone (IIA, 2014).

\subsection{Leisure}

A German study conducted with 14-92 year olds found that people with higher socioeconomic status have more access to the internet and are more inclined to use its many possibilities and applications (electronic mail, data 
search, purchases) in all age groups. A considerable proportion (9.3\%) reported negative consequences, such as excessive surfing, health compromises, as well as giving up leisure activities, entertainment, and social life (Beutel et al., 2011).

Children and teens have almost unlimited access to the web, including technological instruments: computer, cellular phone, and i-pad. Use of technological instruments is becoming an inseparable part of daily life and is almost taken for granted. The internet is an inseparable part of this generation's environment and a place of entertainment, leisure, and means of communication with friends (Harari et al., 2010).

\subsection{Net Spark}

Almost all public schools in the US have internet access on school computers. Since the internet includes a variety of inappropriate material, this is a source of concern for many parents and teachers. In 2000, all public schools had access to the internet, about $98 \%$, and there were accepted use policies (AUPs) and varied technologies such as programs for blocking or filtering internet systems, "honor codes" for students, and supervision by the teaching staff in order to control students' access to inappropriate material. Between $95 \%$ and $100 \%$ of schools of all types took the AUP approach, 94\% reported access to internet surveillance by staff members, $74 \%$ reported that they use internet blocking or filters, and $64 \%$ had "honor codes". These data show that most schools $(91 \%)$ use more than one procedure or technology as part of their policy to maintain the safety of students' surfing at school (Wells and Lewis, 2006).

It is evident that, as in many other countries, in Israel teachers and parents ascribe much significance to supervising students' internet use at school and elsewhere. At present, with the technological developments and the rising use of smartphones, it is not sufficient to supervise websites on school computers; rather, the aim is to ensure students' safe use of their smartphones as well. For this purpose, a program called NetSpark was developed in response to this need.

NetSpark has a dynamic capacity for graphic analysis, enabling it to constitute a filter solution that takes into account the continuous evolution of internet technology. The program uses automatic identification technologies to filter images on the internet, and provides full protection, particularly where there is no text to help decide whether to enable or block content. The "learning engine" examines unfamiliar images, analyzes image components associated with those already classified in NetSpark's image database, and is capable of determining whether the image in its entirety is appropriate and can be approved or must be blocked. The graphics engine can distinguish between images that contain pornographic or adult content (for example, bathing suits worn on the beach, and also bathing suits or underwear sold on the internet, etc.). With more than a $99 \%$ chance of success, this is the most advanced technology on the market. NetSpark's breakthrough filter solutions let users take control of their internet experience, preserve access to the large range of resources on the web, yet protect them from inappropriate content. NetSpark recognizes the enormous value of the internet as a useful tool and a source of knowledge, perceived as an important resource for all members of modern society.

Despite its enormous potential, the internet also involves risks. Parents and teachers are increasingly searching for ways to protect their children and themselves from websites with inappropriate contents. Workplaces, schools, and other public places are also seeking a new solution that will enforce an accepted use policy and prevent abuse of the public internet access they provide. NetSpark provides this solution. The company's unique technological approach examines automatically and filters content requests, evaluation of content on a page, in real time, and removes all inappropriate text or picture components before supplying the necessary content. This leaves a fully reconstructed page. NetSpark offers a unique filter that is capable of providing such results, and provides access to the maximal amount of content possible.

NetSpark provides several services - supervision of graphics, filtering - avoiding exposure to undesirable content without blocking the entire internet page, filtering content in real time - supervision and filtering of the page during use. Safety check of a website: The quantity of pornographic and inappropriate content found on safe websites is enormous, and should not be disregarded. The program gives users the ability to include filters, in order to ensure users' safety and access to appropriate content on the website, social media solutions - when coping with current-day problems (cyberbullying, hunters, and sexting), NetSpark enables supervision of profiles on social networks, chat messages, and blogs, in order to identify and respond to inappropriate content (NetSpark).

The purpose of this study was to examine whether there is a correlation between the existence of the NetSpark program on smartphones and utilization of leisure time among students who use or do not use the program. The hypothesis was that students who use the program will be more inclined to occupy themselves with activities unrelated to the internet, such as spending time with friends, physical activity, etc., and when the program is not used they will be more inclined to stay at home and use the internet. We focused on female students at the Ofra religious high school for girls, where the program was installed on students' cellphones by the school administration, and students of the same school on whose phones the program was not installed. We also examined the time devoted to internet use on smartphones after installation of the NetSpark program. In addition, we examined whether the change enforced by the school by installing the program changed students' use habits after school hours. Finally, we also sought to explore whether the variable of religiosity has an effect on students' leisure activities.

The research hypotheses were:

1. A difference would be found in cellphone use patterns, such that students who use the program will show less use of applications than students who do not use the program.

2. An association would be found between installation of the program that blocks inappropriate content and utilization of leisure time. Among students who use the program we will see varied leisure activities unrelated to internet use and for a longer period of time throughout the day than among students who do not use the program.

3. An association would be found between internet use habits and religiosity. The higher one's religiosity the lower the internet use, and the lower one's religiosity the higher the internet use. 
4. An association would be found between religiosity and utilization of leisure time. Namely, the higher one's religiosity, the more leisure time will be devoted to activities other than internet use, and the lower one's religiosity the higher the internet use and the less additional activities.

\section{Method}

\subsection{Participants}

The study included $1209^{\text {th }}-12^{\text {th }}$ grade students at the Ofra high school for girls. Ages ranged from 14 to 18 (mean - 16). Half the respondents (60) were students on whose phone the NetSpark program had been installed, and half the students (60) were those who had not had the program installed on their phone.

\subsection{Tools}

The study utilized three questionnaires: a sociodemographic questionnaire, a questionnaire on smartphone use patterns, and a questionnaire on utilization of leisure time. Details of the questionnaires:

1. Sociodemographic questionnaire - a personal information questionnaire for respondents: sex, year of birth, religiosity.

2. Questionnaire on smartphone use patterns -

- The first part of this questionnaire included three questions: was the program installed on the user's phone, years of cellular phone use, and average duration of use per day.

- The questionnaire included 9 items that check to what extent cellular applications are used. This questionnaire was taken from a study by David and Tzipora (2011). The original questionnaire had 7 items, but since more cellular applications frequently used by teens were added in the years since composition of the questionnaire, the researchers added two items: use of WhatsApp and use of social networks. The respondents were requested to grade the items on a 5-point Likert scale, from "not at all" (1) to "very much" (5).

- Questionnaire on utilization of leisure time - taken from a study by Cohen and Romi (2015). This is a self-report questionnaire that consists of 27 items, which check how much time respondents devote per day to the leisure activities listed. Items are graded on a scale of 1-5 from "not at all" (1) to "a very significant amount of time" (5).

Table 1 presents the reliability and the descriptive statistics of the research variables:

Table-1. Reliability and descriptive statistics of the research variables (mean, standard deviation, and range)

\begin{tabular}{l|l|l|l|l}
\hline & Reliability & Mean & Standard deviation & Range \\
\hline Use of smartphone applications & 0.67 & 2.72 & 0.67 & $1.43-4.43$ \\
\hline Utilization of leisure time & 0.75 & 2.48 & 0.40 & $1.44-3.56$ \\
\hline Source: Researcher's data &
\end{tabular}

Source: Researcher's data

\subsection{Procedure}

The process of locating respondents was conducted in person at the school, on a voluntary basis, after receiving the approval of the school principal. Completion of the questionnaire was anonymous.

\section{Results}

First, we shall present the distribution of the research variables.

Table-2. Descriptive statistics of the research variables (frequency distribution)

\begin{tabular}{|c|c|c|c|}
\hline Program installed & No & 55 & 43.0 \\
\hline \multirow{3}{*}{ Duration of cellular phone use } & One to three years & 90 & 70.3 \\
\hline & More than nine years & 4 & 3.1 \\
\hline & Less than one year & 10 & 7.8 \\
\hline \multirow{3}{*}{ Time spent on the internet } & One or two hours & 36 & 28.1 \\
\hline & Two or three hours & 35 & 27.3 \\
\hline & Five hours or more & 22 & 17.2 \\
\hline
\end{tabular}

Source: Researcher's data and analysis

According to the first research hypothesis, a difference would be found between respondents in use of cellular applications, use of cellular phones, and daily duration of internet use, by use of the program. In order to examine the research hypothesis, t-tests for independent samples were performed. Table 3 describes the results of the analyses.

Table-3. Differences in use of cellular applications, use of cellular phones, daily duration of internet use, between respondents with NetSpark installed/not installed

\begin{tabular}{|c|c|c|c|c|c|}
\hline & & Program used & & ogram not used & $t(126)$ \\
\hline & $\bar{M}$ & $\mathrm{SD}$ & $\bar{M}$ & $\mathrm{SD}$ & \\
\hline Use of cellular applications & 2.67 & 0.69 & 2.80 & 0.64 & -1.06 \\
\hline Length of cellular phone use & 2.12 & 0.57 & 2.23 & 0.63 & -1.05 \\
\hline Daily duration of internet use & 2.39 & 1.08 & 2.25 & 1.04 & 0.74 \\
\hline
\end{tabular}


The research findings show a significant difference between respondents in the use of computer applications, use of cellular phones, and daily duration of internet use, by use of the program.

According to the second research hypothesis, a difference would be found in utilization of leisure time between respondents by program use. In order to examine the research hypothesis, a t-test for independent samples was conducted, $t(126)=0.70, \mathrm{p}>.05$. No significant difference was found in the utilization of leisure time between respondents who used the program $(\mathrm{M}=2.50, \mathrm{SD}=0.44)$ and who did not use the program $(\mathrm{M}=2.45, \mathrm{SD}=0.35)$.

According to the third research hypothesis, a joint effect of religiosity and of the NetSpark program would be found on the duration of cellular phone use. For this purpose, a two-way analysis of variance was conducted. As evident from Table 4, use of the program was found to have no main effect, $F(1,124)=0.08$, p >.05. No main effect was found for religiosity either, $F(1,124)=0.56, \mathrm{p}>.05$.

Table-4. Mean and standard deviation of length of cellular phone use by religiosity and NetSpark program

\begin{tabular}{l|r|r|r|r|r|r}
\hline & \multicolumn{2}{|c|}{ Program used } & \multicolumn{2}{r}{ Program not used } & \multicolumn{3}{r}{ Total } \\
\hline & $\mathbf{M}$ & $\mathbf{S D}$ & $\mathbf{M}$ & $\mathbf{S D}$ & $\mathbf{M}$ & SD \\
\hline Respondents with low religiosity & 2.33 & 0.51 & 2.33 & 0.57 & 2.33 & 0.50 \\
\hline Respondents with high religiosity & 2.10 & 0.58 & 2.23 & 0.64 & 2.15 & 0.61 \\
\hline Total & 2.12 & 0.57 & 2.23 & 0.63 & & \\
\hline Source: Researcher's data and analysis & \multicolumn{6}{|l}{}
\end{tabular}

Source: Researcher's data and analysis

According to the fourth research hypothesis, a joint effect of religiosity and of program use would be found on the daily duration of internet use. For this purpose, a two-way analysis of variance was conducted. As evident from Table 5, there is no main effect of the NetSpark program, $F(1,124)=0.59$, p >.05. No main effect was found for religiosity either, $F(1,124)=2.70, \mathrm{p}>.05$.

Table-5. Mean and standard deviation of daily hours of internet use by religiosity and NetSpark

\begin{tabular}{l|l|l|l|l|l|c}
\hline & \multicolumn{2}{|l|}{ Program used } & \multicolumn{2}{l}{ Program not used } & \multicolumn{2}{l}{ Total } \\
\hline & M & SD & M & SD & M & SD \\
\hline Respondents with low religiosity & 3.16 & 1.32 & 2.66 & 1.52 & 3.00 & 1.32 \\
\hline Respondents with high religiosity & 2.32 & 1.05 & 2.23 & 1.02 & 2.28 & 1.03 \\
\hline Total & 2.39 & 1.08 & 2.25 & 1.04 & & \\
\hline Source: Researcher's data and analysis
\end{tabular}

Source: Researcher's data and analysis

A two-way analysis of variance was conducted. As evident from Table 6, no main effect was found for use of the program, $F(1,124)=1.13$, p >.05, however a main effect was found for religiosity, $F(1,124)=18.91, \mathrm{p}>.001$.

Table-6. Mean and standard deviation of cellular application use by religiosity and NetSpark

\begin{tabular}{|c|c|c|c|c|c|c|}
\hline & \multicolumn{2}{|c|}{ NetSpark program installed } & \multicolumn{2}{|c|}{ NetSpark program not installed } & \multicolumn{2}{|c|}{ Total } \\
\hline & $\bar{M}$ & $\mathrm{SD}$ & $\mathrm{M}$ & $\mathrm{SD}$ & $\bar{M}$ & $\overline{\mathrm{SD}}$ \\
\hline Respondents with low religiosity & 3.50 & 0.70 & 3.85 & 0.49 & 3.61 & 0.63 \\
\hline Respondents with high religiosity & 2.60 & 0.65 & 2.73 & 0.60 & 2.66 & 0.63 \\
\hline Total & 2.67 & 0.69 & 2.80 & 0.64 & & \\
\hline
\end{tabular}

A two-way analysis of variance was conducted. As evident from Table 7, no main effect was found for use of the program, $F(1,124)=0.07$, and no main effect was found for religiosity, $F(1,124)=0, \mathrm{p}>.05$.

Table-7. Mean and standard deviation of utilization of leisure time by religiosity and the NetSpark program

\begin{tabular}{|c|c|c|c|c|c|c|}
\hline & \multicolumn{2}{|c|}{ NetSpark program installed } & \multicolumn{2}{|c|}{ NetSpark program not installed } & \multicolumn{2}{|r|}{ Total } \\
\hline & $\bar{M}$ & $\mathrm{SD}$ & $\mathrm{M}$ & $\mathrm{SD}$ & $\bar{M}$ & $\overline{\mathrm{SD}}$ \\
\hline Respondents with low religiosity & 3.50 & 0.70 & 3.85 & 0.49 & 3.61 & 0.63 \\
\hline Respondents with high religiosity & 2.60 & 0.65 & 2.73 & 0.60 & 2.66 & 0.63 \\
\hline Total & 2.67 & 0.69 & 2.80 & 0.64 & & \\
\hline
\end{tabular}

Source: Researcher's data and analysis

\section{Conclusion and Discussion}

The purpose of the current study was to examine whether there is an association between installing on students' smartphones a program blocking internet access and utilization of their leisure time. The research findings show no significant association between the installation of an internet blocking program and use of cellular applications and utilization of leisure time among students who used or did not use the program, nor was a difference between respondents found on these variables by their self-reported religiosity.

The research findings illuminate the subject of internet use and utilization of leisure time. Evidently, although most of the hypotheses were found insignificant, higher use of cellular applications was found among respondents with low religiosity than among respondents with high religiosity. This shows that less religious respondents make greater use of their smartphones, spend more time online, and devote more time to this than students who reported being more religious. Religiosity may have a certain effect on respondents' choice to invest more or less time in use of their smartphones.

The significance of the finding for teachers and educators who seek to use some external program to block internet, even among this special sector, is that internet use must be dealt with through other means. The research findings raise doubts as to the ability to supervise by means of blocking and raise thoughts on the need to teach students skills for coping in the digital world. The religious school system must adapt religious life to the changing world in the field of leisure as well, even though the penetration of modern values into the religious world arouses a sense of "discomfort" in the religious school system. 
Leisure culture has become a major topic of discussion throughout the observant Jewish world, with parents, educators, and rabbis busy with the question of how consumption of leisure culture should be treated, to begin with (in a planned and initiated manner) or in retrospect (not planned and initiated, when teens are "enticed" to follow the temptations of leisure and the supply of leisure) (Eldar, 2011). Managing leisure in the current era is a significant challenge both for the public in general and for the traditional public in particular. The desire to adapt religious life to modern times and the attempt to continue checking in the traditional way constitute a real challenge for educators everywhere.

In 2011, an entire issue of the journal "Bemahshava Tehila" was dedicated to leisure culture, dealing with how to educate towards correct utilization of the leisure culture in religious society. A wide range of educators and leaders from religious public schools presented their impressions with regard to the dangers posed by leisure and discussed how leisure should be appropriately managed. The discussion indicates that despite growing closer to the modern world, religious society is still subject to numerous restrictions concerning the proper consumption and perception of leisure culture. Educators and leaders in the national religious school system are united in their opinion against leisure as a value per se, and object to the transformation of leisure into a "new God", as may be happening in modern society. In their opinion, leisure in traditional society cannot constitute a value in its own right rather only a means to a larger purpose. In this manner, religious society is required to proceed with care as it must teach its students to be capable of choosing, exercising self-control and assuming personal responsibility for their actions (Eldar, 2011). This is precisely because they live in a setting circumscribed by Jewish law. The educational challenge grows and intensifies in a world that is full of possibilities and temptations, one where "the student's access to the world, with all its challenges and dangers, is greater than ever before, even when the student is at home, in his room, facing the computer and the internet" (ibid., p. 11).

From the educational aspect, there is a concern of the negative potential of leisure and the fact that we have not yet managed to find ways of overcoming the dangers of leisure (violence, alcohol abuse, gambling, etc.). It is necessary to address the consumption of leisure that is mainly technology-based, including products that have entered homes and schools - and there is no way of escaping their influence, rather only of coping. Experience shows that it is possible to bridge the gaps between modernity and tradition. Leisure activities in religioustraditional society make it possible, on one hand, to enjoy leisure activities, and on the other to create a regulating and supervising setting. In this way, the possibility that leisure will become a sanctified value in its own right is avoided. Religious society puts special emphasis on the restriction (for example: Rimon Internet, restricted cellular tracks) of leisure consumption, for fear of a slippery slope. Indeed, in today's world, there are many temptations and sometimes there is an experience of "brainwashing" by messages conveyed in different ways, insinuating that we need more and more of everything. Precisely in such a world, the religious environment is an anchor that makes it possible to put the temptations, leisure culture, in proportion, and reminds one that our inner world is best filled not from the outside but rather by contact with the Creator. The purpose of educators in modern times is to preserve the old world of values within a new route, following the principle of "the old shall be renewed and the new, sanctified" (Rav Kook, Letters of the Re'ayah, 3:216), which implies a need to merge the best of Jewish tradition with general modern culture, based on a comprehensive ethical outlook.

\section{References}

Almog, O., 2004. Predah misrulik: Shinui arachim baelitah hayisre'elit [Farewell to Srulik: Changing Values among the Israeli Elite]. Haifa, Israel: University of Haifa Press \& Zmora-Bitan, 1.

Bar-Lev, M., 1977. Yeshiva high school graduates in Israel: Between tradition and innovation. Ph.D. Thesis, Bar-ILan University, Ramat Gan.

Bar-Lev, M., 1981. Changes in the leisure patterns of religious jews. Leisure and recreation culture in Israel. Ed. Aori. Liptzin. Tel Aviv: Cherikover. pp: $21-26$

Ben Zvi, L., 1994. Knitted kippa: Life style and subcultural of the young. Bisdeh Hemed, 38(3-4): 73-84.

Beutel, M.E., E. Brähler, H. Glaesmer, D.J. Kuss, K. Wölfling and K.W. Müller, 2011. Regular and problematic leisure-time internet use in the community: Results from a German population-based survey. Cyberpsychology, Behavior, and Social Networking, 14(5): 291296. View at Google Scholar | View at Publisher

Brandes, Y., 2011. Leisure culture between the commanded and the permitted. Bemahshava Tehila. Israel: Reshet Amit Publishing, 72: 1838.

Cohen, E.H. and S. Romi, 2015. Leisure among youth in Israel: Informal education, school climate, violence, risk of dropout and well-being. Ramat Gan: Bar Ilan University.

David and Tzipora, 2011. Use of cellular telephones: Jewish teens versus bedouin teens in Israel. Beer Sheva: Ben Gurion University.

Eldar, A., 2011 . Opening words of the director general. Bemahshava Tehila. Israel: Reshet Amit Publishing, 72: 11-13.

Gopes, D., 2011. Why is it so hard for us? Leisure in modern society. Bemahshava Tehila. Israel: Reshet Amit Publishing, 72 : 44-47.

Harari, L., M. Vidislavsky and D. Shemesh, 2010. The way to a safe space on the internet. Journal for Promoting Leadership, Professionalism, and Excellence in the School Administration in Elementary Education. Jerusalem: Ministry of Education, 28: 117.

Israel Internet Association, 2014. Retrieved from https://www.isoc.org.il/sts-data/11063 [Accessed 1 August 2017].

Lenhart, A., 2015. Teens, social media \& technology overview. Pew Research Center. Retrieved from http://www.pewinternet.org/2015/04/09/teens-social-media-technology-2015/. http://www.netspark.com/?a=technologies/graphic_inspection.

Rafaeli, S., Y. Ariel and M. Katzman, 2010. Online youth: Internet use and purchase patterns. Jerusalem: Ministry of Trade and Employment.

Sasson, H., R. Erez and Z. Elgali, 2011. Risks on the web: Adolescents' perceptions and coping mechanisms. The 4th Knowledge Cities World Summit. pp: 350-356.

Shapira, N.A., T.D. Goldsmith, P.E. Keck, U.M. Khosla and S.L. McElroy, 2000. Psychiatric features of individuals with problematic internet use. Journal of Affective Disorders, 57(1): 267-272. View at Google Scholar | View at Publisher

Shapira, N.A., M.C. Lessig, T.D. Goldsmith, S.T. Szabo, M. Lazoritz, M.S. Gold and D.J. Stein, 2003. Problematic internet use: Proposed classification and diagnostic criteria. Depression and Anxiety, 17(4): 207-2 16. View at Google Scholar |View at Publisher

Wells, J. and L. Lewis, 2006. Internet access in US public schools and classrooms: 1994-2005. (NCES 2007-020). National Center for Education Statistics. Washington, DC: US Department of Education. 